\title{
Desplazamiento horizontal límite en arcos de fábrica
}

\section{Limit horizontal displacement of masonry arch on spreading supports}

Lore Mutiloa $^{(*)}$, Adrián Guamán ${ }^{(* *)}$, Javier León ${ }^{(* *)}$, Leonardo Todisco ${ }^{(* * *)}$

\section{RESUMEN}

El nivel de seguridad de los arcos de fábrica se ve muy afectado por posibles desplazamientos que puedan producirse en sus arranques. Tales desplazamientos generan un incremento o una disminución de los empujes horizontales. Este proceso, que se retroalimenta, puede disminuir sensiblemente le seguridad de las estructuras y incluso llevarlas al colapso. A pesar de la importancia de este fenómeno, la bibliografía al respecto es escasa y principalmente enfocada a arcos de directriz circular. En este artículo se presenta una herramienta desarrollada para determinar el desplazamiento límite horizontal hacia el exterior de arcos de cualquier directriz considerando o no la existencia de un relleno. Además, se realiza un análisis paramétrico para estudiar el efecto de un desplazamiento impuesto para diferentes geometrías y varias relaciones canto/radio o canto/luz, lo cual lleva a la construcción de gráficas muy útiles para la práctica profesional. Finalmente, se aplica la metodología a un ejemplo real.

Palabras clave: Desplazamiento límite; mecanismo de colapso; arcos de fábrica; línea de empujes; rótulas.

\section{ABSTRACT}

The safety of masonry arches is heavily related to possible displacements that may occur at supports. These movements can be outwards or inwards, with a consequent, respectively, increase or decrease of the horizontal thrust. This process may strongly reduce the safety of a masonry structure, and even lead to its collapse. Despite the importance of this phenomenon, the bibliography is scarce and mainly focused on arches with circular shapes. This paper presents the development of a tool to estimate the outward horizontal support movements necessary to cause collapse for masonry arches of any shape considering, if needed, the existence of a backfill. Next, this work illustrates a parametric analysis to study the effect of support displacements on masonry arches with different shapes and several thickness/span ratios. This results in the construction of very useful plots for the professional practice. Finally, the methodology is applied to a real case of study.

Keywords: Limit displacement; masonry arches; collapse mechanism; spreading supports.

(*) Ingeniera de caminos, canales y puertos. Ingeniera de Proyecto. FHECOR Ingenieros Consultores, Madrid (España)

(**) Ing. Civil. Alumno de máster. Universidad Politécnica de Madrid, Madrid (España)

$\left.{ }^{* * *}\right)$ Dr. Ing. de caminos, canales y puertos. Profesor Titular. Universidad Politécnica de Madrid, Madrid (España)

(****) Dr. Ing. de caminos, canales y puertos. Profesor Ayudante Doctor. Universidad Politécnica de Madrid, Madrid (España)

Persona de contacto/Corresponding author: leonardo.todisco@upm.es (L. Todisco)

ORCID: https://orcid.org/oooo-0002-5378-080o (L. Mutiloa); https://orcid.org/oooo-0oo3-1127-0105 (A. Guamán); https:// orcid.org/oooo-0003-1284-2216 (J. León); http://orcid.org/oooo-00o3-1077-4942 (L. Todisco)

Cómo citar este artículo/Citation: Lore Mutiloa, Adrián Guamán, Javier León, Leonardo Todisco (2021). Desplazamiento horizontal límite en arcos de fábrica. Informes de la Construcción, 73(564): e417. https://doi.org/10.3989/ic.82665

Copyright: (C) 2021 CSIC. Este es un artículo de acceso abierto distribuido bajo los términos de la licencia de uso y distribución Creative Commons Reconocimiento 4.0 Internacional (CC BY 4.0). 


\section{INTRODUCCIÓN}

\subsection{Motivación}

La evaluación de la seguridad de una estructura de fábrica es una tarea compleja debido a la naturaleza del material constituyente (anisótropo, no homogéneo y de propiedades mecánicas de difícil estimación fiable) y a un comportamiento mecánico y geométrico marcadamente no lineal. El nivel de seguridad de una estructura de fábrica, una catedral o un puente, por ejemplo, está íntimamente relacionado con la capacidad de sus arcos y bóvedas de resistir los desplazamientos o giros que puedan producirse en sus arranques, hasta el punto de que puede llegarse al colapso, a igualdad de cargas solicitantes, si tales movimientos superan unos ciertos umbrales. No se trata solamente de alcanzar el equilibrio estático de fuerzas para una geometría inicial, sino de asegurar que la deformada de la estructura no provoque efectos de segundo orden que acaben por atentar contra el equilibrio global de la estructura.

A una rotación o un desplazamiento de los pilares o de los muros que soportan un arco corresponde una variación de la geometría del mismo. Baste pensar que, en un pilar de 10 metros de altura, una rotación de sólo un grado respecto a la vertical genera un incremento de luz en el arco soportado por él de unos $34 \mathrm{~cm}$ ( $17 \mathrm{~cm}$ por cada lado) (1).

Al producirse tales movimientos en los arranques, el arco de fábrica cambia su geometría de partida, abriéndose las juntas entre dovelas en determinadas posiciones, con comportamiento dúctil, adaptándose a la nueva condición de contorno. Los desplazamientos de los arranques pueden ser hacia el exterior o hacia el interior, generando respectivamente un rebajamiento o un incremento de la flecha, lo cual conlleva, respectivamente, un incremento o disminución de los empujes horizontales. Este proceso, que se retroalimenta, puede disminuir sensiblemente le seguridad de las estructuras de fábrica, y hasta puede llevarlas al colapso, como se ha anticipado.

Es el caso, muy extendido, de bóvedas pesadas (no sólo las románicas) en las que el empuje ha deformado muros y pilas hacia el exterior, teniéndose que acudir a recursos como el atirantamiento (Figura 1). Aunque la deformada de pila y bóveda es perceptible, no siempre lo son las juntas abiertas entre dovelas, puesto que los sucesivos trabajos de restauración han ido tapándolas y, en parte, enmascarando el problema. $\mathrm{Al}$ tratarse de un fenómeno no lineal geométrico, se requiere un análisis paso a paso para poder deducir la geometría final antes del colapso.

A pesar de la importancia de este fenómeno, la bibliografía al respecto es escasa y está principalmente dirigida a arcos de directriz circular. En definitiva, este fenómeno no ha sido suficientemente estudiado para casos de arcos con geometría cualquiera. Tampoco se ha considerado el efecto del relleno que suele existir en el trasdós.

\subsection{Objetivos}

El principal objetivo de este artículo es describir el método seguido, junto con la herramienta asociada, para hallar el desplazamiento límite horizontal, hacia el exterior, de arcos de fábrica de cualquier directriz.

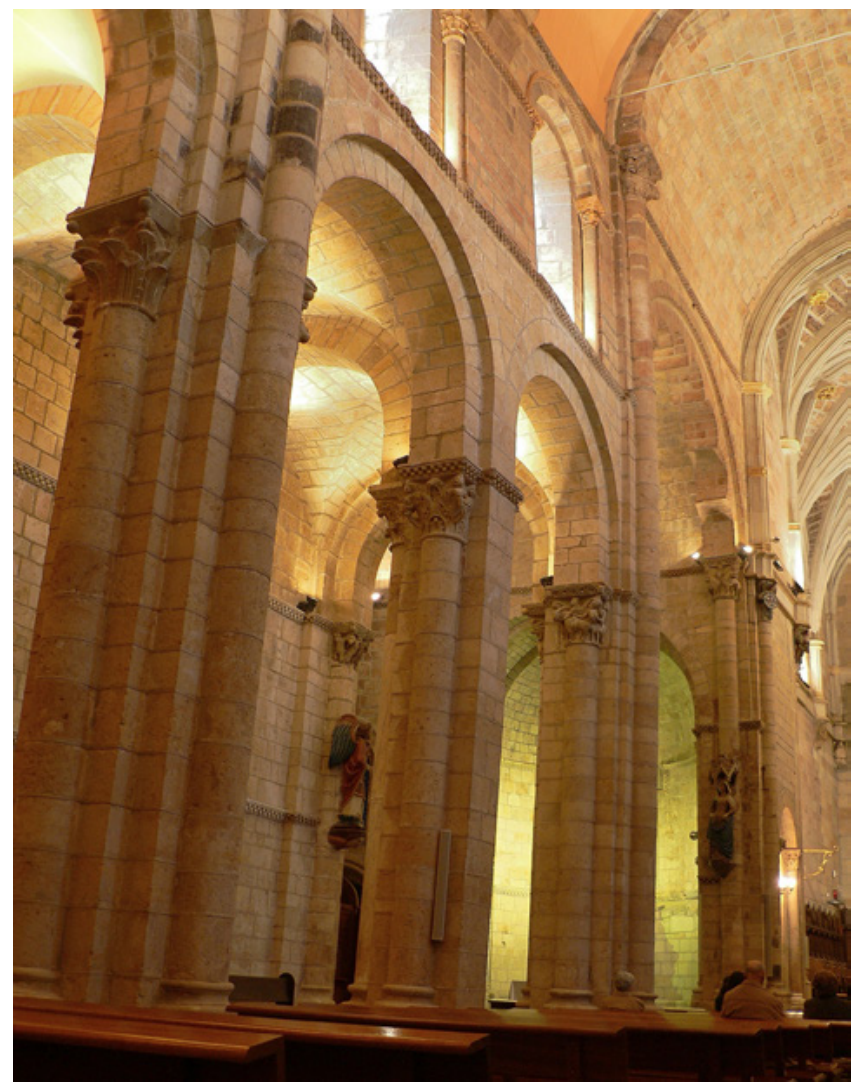

Figura 1. Deformada de muros y pilares que inducen, a su vez, deformaciones en la bóveda alta, primigeniamente de medio punto (foto de los autores).

Este estudio incluye también la consideración del relleno granular, analizado paramétricamente para diferentes alturas. Los resultados representan gráficamente el máximo incremento de luz que los arcos de fábrica pueden experimentar en función de su geometría y relaciones canónicas. Estas gráficas ayudan en la práctica profesional para obtener los desplazamientos máximos esperables sin necesidad de realizar un análisis no lineal geométrico. Finalmente, se presenta la aplicación de lo expuesto a un caso práctico real.

\subsection{Organización del artículo}

En primer lugar, se presenta una breve síntesis del estado del conocimiento de arcos de fábrica sobre soportes con desplazamientos impuestos. Se describe después la metodología utilizada para el análisis de desplazamientos límite en los arcos de fábrica, con la herramienta que posibilita el cálculo. A continuación, se presentan los resultados de los análisis realizados. Tras exponer el caso práctico analizado, se presentan finalmente las conclusiones y se plantean futuras investigaciones.

\section{ESTADO DE LA CUESTIÓN}

El análisis de una estructura de fábrica se basa en las líneas de empuje a partir de las siguientes hipótesis (2-4):

1. La fábrica tiene una resistencia a compresión infinita.

2. La fábrica tiene una resistencia a tracción nula.

3. La fábrica tiene una resistencia a cortante infinita.

Estas tres hipótesis se pueden resumir diciendo que la fábrica se comporta como un material perfectamente plástico en 
tracción y perfectamente rígido en compresión y cortante. Las hipótesis 1 y 3 se basan en el hecho que, generalmente, el nivel tensional al que está sometida la fábrica es mucho menor que su resistencia a compresión y cortante. No obstante, suponer infinitas las capacidades resistentes de los materiales puede quedar en algunos casos del lado de la inseguridad (5). En otras palabras, encontrar una situación equilibrada (una línea de empujes situada en el interior de la fábrica) es condición necesaria, pero no suficiente, para pronunciarse acerca de la seguridad de la estructura. Luego, hay que controlar que las tensiones normales o tangenciales, o ambas combinadas, no sobrepasan la envolvente de rotura, definida a partir de la expresión de Mann-Müller (6) y, por lo tanto, confirmar las hipótesis hechas previamente. Además, debe considerarse que, aunque la resistencia de la fábrica a corte sea baja, el aparejo dispuesto en arcos y bóvedas hace que en la mayoría de las ocasiones la línea de empujes sea casi ortogonal a la superficie de deslizamiento (tendeles) y, por lo tanto, los esfuerzos tangenciales sean pequeños. Finalmente, la hipótesis 2 está del lado de la seguridad y equivale a considerar despreciables tanto la resistencia a tracción del mortero como la adherencia de éstas a las piezas.

El análisis basado en línea de empujes se enmarca en los teoremas de los límites inferior y superior de la teoría de la plasticidad. Así, a los efectos de este artículo, cabe recordar que el teorema del límite inferior, aplicado a las estructuras de fábrica, se puede enunciar de la siguiente manera: "Si es posible definir una línea de empujes que se encuentre en equilibrio bajo la acción de las cargas exteriores, y que esté contenida en el espesor de las piezas, la estructura será estable para esas cargas, que serán inferiores a las de colapso”.

Un arco de fábrica cuyos arranques se desplazan horizontalmente, que es el objeto de este estudio, puede alcanzar el colapso de dos maneras: por un mecanismo simétrico de cinco rótulas (mecanismo similar al arco de espesor mínimo) o un mecanismo de tres rótulas alineadas si el canto es suficientemente grande (7). Para la mayoría de los arcos, el fallo estará gobernado por el mecanismo de colapso de cinco rótulas, siendo la parte central del arco un arco de tres rótulas que se deforma para acomodarse al cambio de geometría. Se trata de un problema no lineal geométrico porque, a medida que la luz aumenta, la ubicación de la rótula del intradós puede cambiar de posición y moverse hacia la clave del arco.

Un estudio relevante y pionero sobre la cuestión fue el de Ochsendorf (1), del cual se reproduce la Figura 2, que pone de manifiesto cómo un movimiento hacia afuera de un arranque, el derecho en este caso, provoca la formación de tres rótulas (A, B y C), dos de las cuales se ubican en el intradós (A y C) y una en el trasdós (B). Una vez conocida la ubicación de estas rótulas es posible determinar el empuje horizontal asociado a esta situación. Si el desplazamiento impuesto sigue aumentando, las piezas en que se ha dividido el arco se desplazarán y rotarán de manera acorde a la cinemática de sólido rígido. La clave del arco (B) desciende y el empuje aumenta debido al cambio de la geometría. Es importante mencionar que, aunque se vaya incrementando el desplazamiento, la componente vertical de la reacción permanecerá constante, mientras que la componente horizontal aumentará (1).

En el mismo trabajo, Ochsendorf (1) analizó una serie de arcos circulares para determinar el desplazamiento horizontal

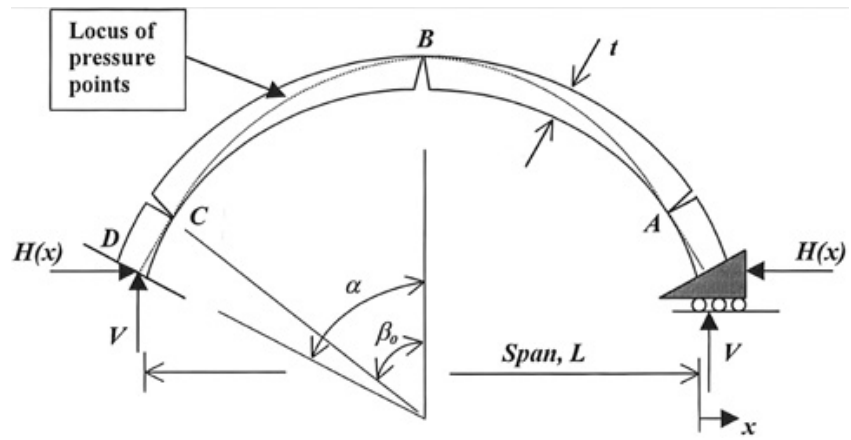

Figura 2. Arco circular con apoyo derecho desplazable (1).

máximo antes de que se produjera el colapso. Se estudiaron arcos circulares con ángulos de $80^{\circ}$ a $180^{\circ}\left(\alpha=40^{\circ}\right.$ a $\left.90^{\circ}\right)$ y con diferentes relaciones de espesor/radio. Los valores correspondientes al incremento de luz que ocasionan el colapso se presentan en la Figura 3 en tanto por ciento respecto de la luz inicial. Se advierte una tendencia casi lineal para diferentes relaciones de espesor/radio. Cada curva comienza con la mínima relación de espesor-radio, y el desplazamiento límite se calcula para valores crecientes de espesores.

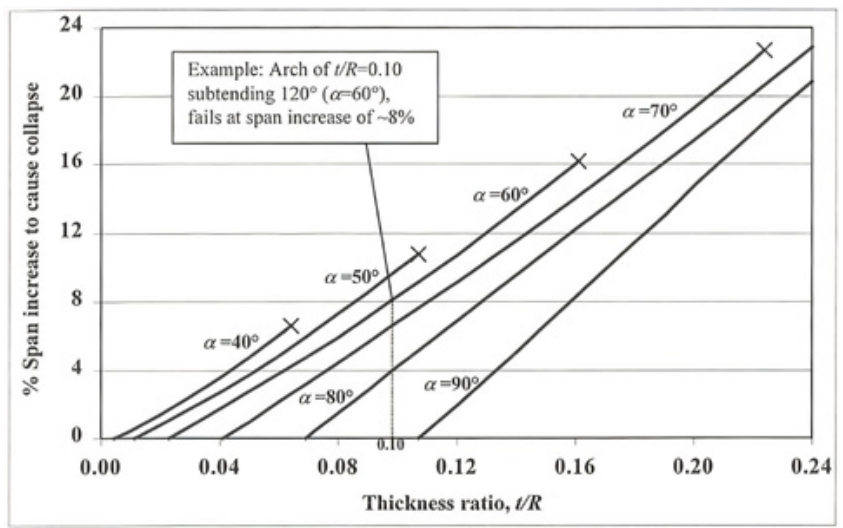

Figura 3. Máximo incremento de luz antes del colapso para arcos circulares de espesor-radio variable (1).

Más recientemente, otros investigadores han realizado estudios sobre desplazamientos impuestos horizontales aplicados a arcos circulares (8), arcos elípticos (9), arcos apuntados (10), bóvedas de directriz circulares (11), bóvedas de crucería (12) e incluso combinando desplazamientos verticales y horizontales (13-15). Para corroborar los resultados numéricos se han realizado ensayos a escala (16) y se han analizado ejemplos reales (17). Todas estas investigaciones recientes están limitadas a arcos cuyos intradós y trasdós pueden expresarse a través de formulaciones analíticas, pero la experiencia ha puesto de manifiesto que las geometrías de los arcos son mucho más variadas, especialmente si se deforman. Además, en los estudios anteriores no se considera la muy relevante contribución de los rellenos en la estabilidad de estas estructuras. Estas son las razones por las cuales se ha realizado el estudio que se presenta en este artículo.

\section{METODOLOGÍA}

El primer paso consiste en definir la geometría del arco de estudio que, en general, está delimitada por las curvas de trasdós e intradós que, con este método, pueden ser cualesquiera. Para describir el procedimiento se emplea un arco de directriz circular. De manera análoga al trabajo de Ochsendorf (1), se definen el radio $R$, el espesor $e$ ( $t$ en (1)) y el valor de án- 
gulo $\alpha$, siendo $\alpha$ el semi-ángulo que abarca el arco (Figura 4). Como se verá más adelante, el empleo de un arco de directriz circular es útil para comparar los resultados con los de las referencias bibliográficas.

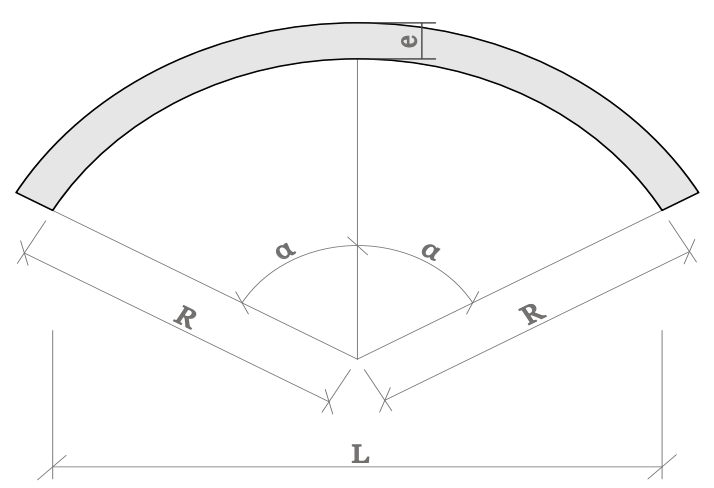

Figura 4. Geometría arco directriz circular.

Una vez definida la geometría, es necesario realizar una discretización ya sea con "dovelas" verticales, lo que comporta un error aceptable (18), o bien con "dovelas" con cortes perpendiculares a la directriz del arco, lo cual se asemeja más al aparejo real. El siguiente paso consiste en calcular el área y, consecuentemente, el peso de cada dovela. A continuación, por simple equilibrio, se calculan los valores de las componentes verticales de las reacciones (constantes a lo largo de todo el proceso), y de las horizontales. Al tratarse de un problema hiperestático (hay infinitas soluciones), hay que tantear varias posiciones de la línea de empujes variando el valor de la componente horizontal de la reacción y modificando también el punto de arranque de la línea de empuje. Obtenidas todas las posibles líneas de empuje compatibles con la estructura (líneas contenidas en el contorno de la pieza), se selecciona aquella que lleve asociado el mínimo empuje horizontal; esto es, la línea contenida dentro del arco y que genere tres rótulas, dos en el intradós y una en el extradós (Figura 5-a). En rigor, el criterio a seguir sería el de buscar la línea de empujes asociada a la mínima energía del sistema, como se hace en (19). Sin embargo, para facilitar las cosas, se utiliza el criterio del mínimo empuje, más sencillo y suficientemente aproximado, como demuestran los modelos físicos observados.

A continuación, se impone un desplazamiento y se dibuja la nueva geometría de acuerdo con la cinemática de los sólidos rígidos A1, A2, A3 y A4 (Figura 5-b) delimitados por las rótulas. El sólido A1 permanece fijo (convencionalmente, porque el problema es relativo al movimiento entre apoyos), el sólido A4 se desplaza horizontalmente, paralelamente a sí mismo, y los sólidos A2 y A3 rotan, haciendo que el arco adopte el estado deformado de la figura 3-c. Con el arco deformado, se obtiene la nueva geometría, presentada en la Figura $5^{-c}$. Una vez dibujada la nueva geometría, el proceso para la obtención de la línea de empujes será análogo al de la geometría sin deformar para la obtención de la línea de empujes asociada al empuje mínimo. En la Figura 5-d, se muestra la secuencia cinemática que siguen las deformadas frente a desplazamientos horizontales impuestos crecientes según la herramienta desarrollada. Se trata de un procedimiento iterativo consistente en imponer sucesivamente desplazamientos horizontales, volviendo a calcular la nueva geometría para cada uno de ellos, con su respectiva línea de empujes, hasta que el número de rótulas sea mayor que tres o que no sea posible encontrar una línea de empujes contenida en el contorno del arco. Cuando esto ocurra, se tantea con un desplazamiento menor hasta encontrar el desplazamiento límite con precisión suficiente.
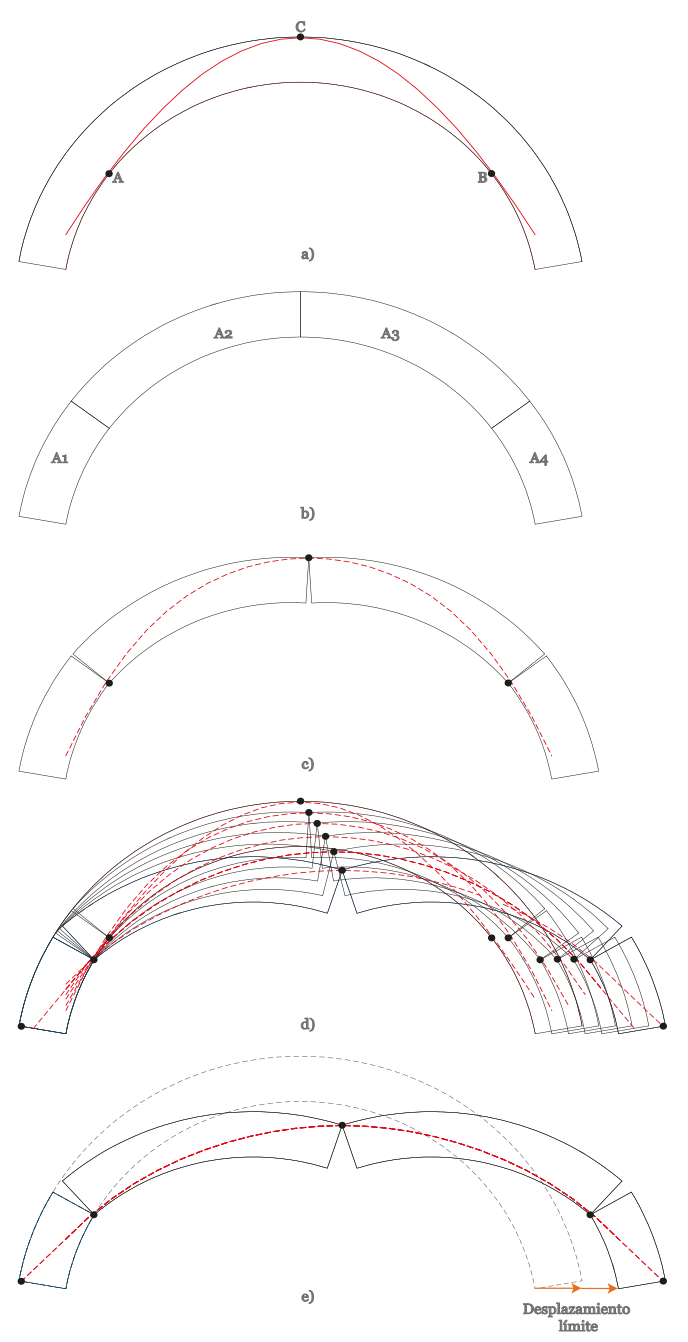

Figura 5. Metodología paso a paso.

Este método se ha implementado en Grasshopper (20), una herramienta de dibujo paramétrico que se ejecuta en la aplicación Rhinoceros 3D (21). Algunos componentes han sido programados específicamente para resolver el problema planteado. La herramienta es libre y se puede obtener enviando un correo electrónico a la persona de contacto del artículo. Las geometrías de intradós y trasdós pueden ser importadas desde AutoCad (22) o bien definirse analíticamente directamente en Grasshopper. La herramienta admite cualquier geometría plana del arco.

\section{RESULTADOS}

\subsection{Arcos de directriz circular}

En primer lugar, se han estudiado seis diferentes configuraciones de arcos de directriz circular, iguales a las empleadas en (1) para, básicamente, validar la metodología. El ángulo $\alpha$ (definido en las figuras 1 y 3)) varía desde $40^{\circ}$ hasta $90^{\circ}$ en cada configuración y, para un mismo ángulo, se han analizado arcos con diferentes relaciones espesor-radio. 


\section{Arcos de directriz circular bajo peso propio}

Con la herramienta se ha obtenido el porcentaje de incremento de luz máxima correspondiente a cada configuración, antes del colapso, variando la relación $e / R$ (figura 6). A igualdad de tipología de arco (mismo radio, mismo material y abarcando el mismo ángulo), cuanto mayor sea el espesor, como resulta esperable, mayor es el desplazamiento límite horizontal.

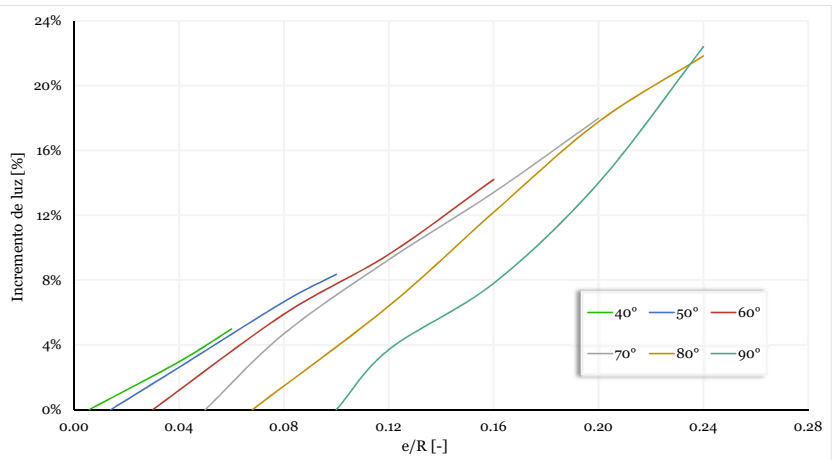

Figura 6. Máximo incremento de luz de arcos de directriz circular al variar de la relación $e / R$.

La gráfica que se obtiene es, de hecho, equivalente a la que se muestra en la Figura 3 obtenida por (1). Los porcentajes de error entre máximo incremento de luz obtenido por el procedimiento descrito anteriormente y el obtenido por (1) no superan el $2 \%$, lo que permite validar la herramienta. Con las gráficas de la Figura 6, es posible obtener líneas de tendencia y determinar ecuaciones que permitan obtener el desplazamiento límite en arcos de directriz circular bajo propio peso, conociendo el valor de la relación $e / R$. Las ecuaciones se presentan a continuación para facilitar su tratamiento digital, conjuntamente con el coeficiente de determinación $\mathrm{r}^{2}$.

Para $\alpha=40^{\circ}$, con valor de $r^{2}=1.00$

$$
\Delta L=2.7108(e / R)^{2}+0.7401(e / R)-0.0044
$$

Para $\alpha=50^{\circ}$, con valor de $r^{2}=0.99$

$$
\Delta L=-1.0434(e / R)^{2}+1.0988(e / R)-0.0155
$$

Para $\alpha=60^{\circ}$, con valor de $r^{2}=0.99$

$$
\Delta L=-0.3364(e / R)^{2}+1.142(e / R)-0.0332
$$

Para $\alpha=70^{\circ}$, con valor de $r^{2}=0.99$

$$
\Delta L=-1.4353(e / R)^{2}+1.5304(e / R)-0.0705
$$

Para $\alpha=80^{\circ}$, con valor de $r^{2}=0.99$

$$
\Delta L=-0.5688(e / R)^{2}+1.4731(e / R)-0.0996
$$

Para $\alpha=90^{\circ}$, con valor de $r^{2}=0.99$

$$
\Delta L=4.0241(e / R)^{2}+0.1715(e / R)-0.0511
$$

Considerando que la relación $e / R$ es solamente válida para los arcos de directriz circular, en la Figura 7 se muestra la gráfica empleando la relación $e / L$, en lugar de $e / R$, donde $L$ es la luz libre entre arranques. El empleo de la luz, en lugar del radio, facilita la comparación de los resultados obtenidos para arcos de directriz circular con arcos de otras formas, tal y como se verá más adelante.

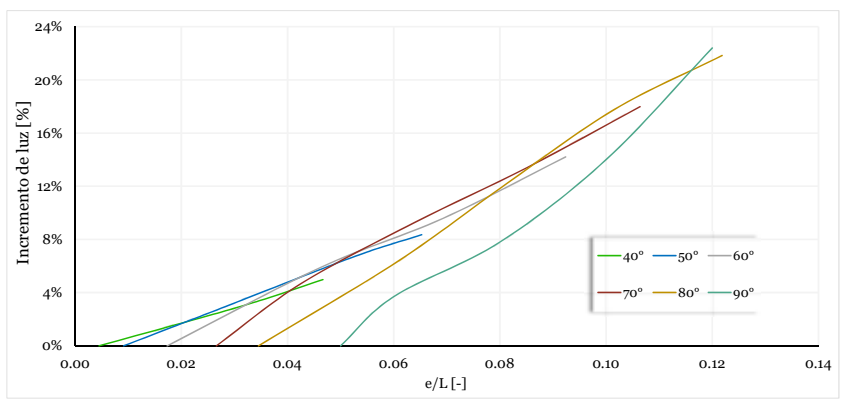

Figura 7. Máximo incremento de luz de arcos de directriz circular al variar de la relación $e / L$.

\section{Arcos de directriz circular con relleno granular}

La existencia de relleno granular es común en la mayoría de los arcos de fábrica existentes y su importancia trascendental en la seguridad estructural ha sido analizada en otros trabajos de este mismo grupo de investigación $(22,23)$. En este artículo se estudia el papel que pueden jugar los rellenos granulares en el desplazamiento límite. En primer lugar, se establecen las posibles alturas del relleno granular: uno, dos y tres tercios de la altura del arco (flecha más espesor, aproximadamente). Las tres posibles configuraciones del relleno granular se muestran en la Figura 8. Una vez establecido un peso específico para el relleno, con la misma herramienta es posible calcular el peso del mismo que se suma al de las dovelas. En el caso de una bóveda, por ejemplo, el terreno que empuja no es indefinido, sino que queda delimitado por el muro que lo contiene. De esta manera pueden definirse diferentes zonas cuyos empujes horizontales son diferentes: por un lado, una zona (la superior) que puede desarrollar empujes similares a los de la teoría clásica (p.e. Coulomb y Rankine), y, por otro, una zona (la inferior) en la cual los empujes son menores por la proximidad del muro (efecto silo). Los estudios realizados demuestran que el empuje que actúa en el trasdós de la bóveda considerando el efecto de paredes próximas es alrededor el 30-40\% del empuje calculado según las teorías clásicas $(19,23)$. En este análisis, del lado de la seguridad en este caso, se considera que el relleno granular no moviliza ningún empuje horizontal en el arco. En la Figura 9 se muestran las gráficas correspondientes a los arcos de directriz circular con relleno granular para cada valor del ángulo $\alpha$. Como en (1), el máximo valor de $e / L$ para el que se dibujan las gráficas se establece como aquél para el que el empuje del arco al colapsar es igual a 5 veces el empuje mínimo. El análisis pone de manifiesto que el aporte del relleno granular es de enorme importancia para el equilibrio del arco; por ejemplo, un arco con un relleno granular hasta dos tercios de altura total (algo muy razonable según (19)), $a=9 O^{\circ}$ y una relación $e / L$ de 0.06 (Figura 9), puede aumentar hasta 4 veces el desplazamiento horizontal límite que tendría sin la presencia del relleno. También se observa que la influencia del relleno granular es tanto mayor cuanto menos rebajado es el arco, siendo máxima en arcos de medio punto.

Es importante señalar que la influencia del relleno rígido puede anularse si éste se separa del trasdós del arco a lo largo 

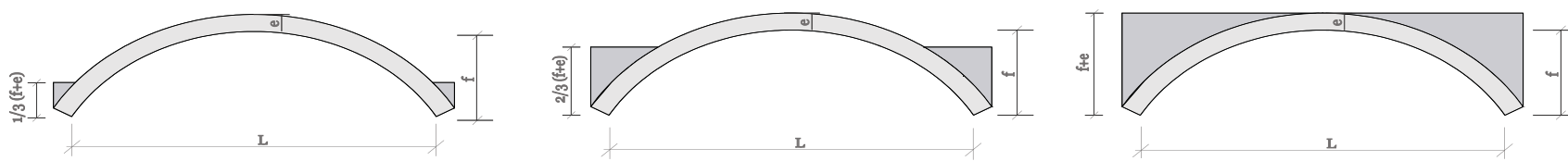

Figura 8. Configuración de arcos circulares con relleno granular hasta una cota de uno, dos y tres tercios de la flecha.
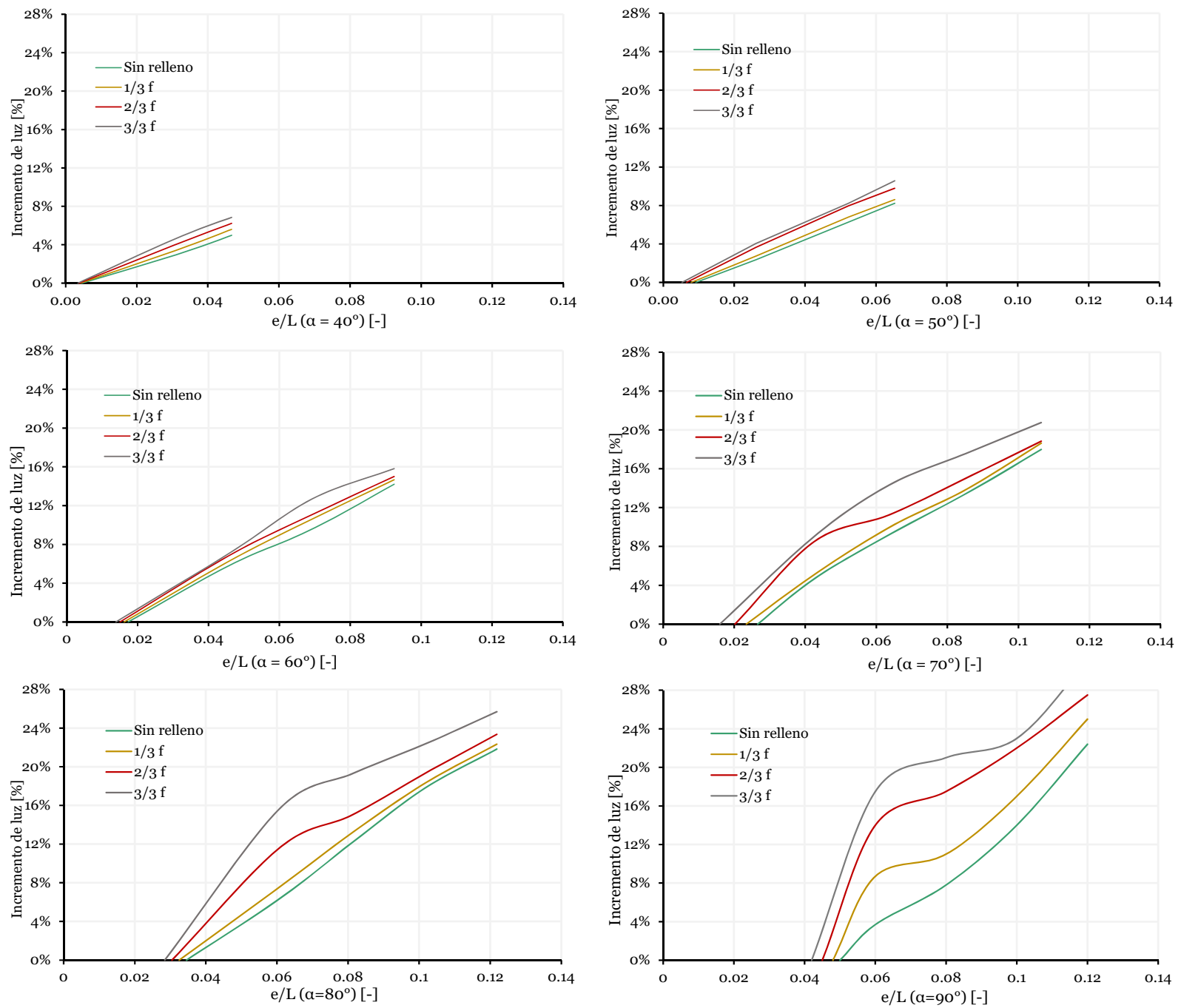

Figura 9. Máximo incremento de luz para arcos de directriz circular con relleno granular.

del proceso cinemático, lo que obliga al técnico a elegir cuidadosamente el valor de $\alpha$ si éste estuviera condicionado por el relleno rígido.

\subsection{Arcos apuntados}

La metodología descrita y la herramienta desarrollada se han aplicado al estudio de los arcos apuntados, tan frecuentes en edificios góticos. Cabe recordar que la bibliografía sobre el desplazamiento horizontal límite en arcos apuntados es muy escasa (10). Siguiendo la misma metodología empleada para los arcos de directriz circular, se establece la altura $h$ que definirá la posición del relleno rígido. En este caso, la relación a emplear será la ratio $e / L$ (Figura 10), siendo $e$ el espesor del arco, con $f$ y $L$ definidos en la Figura 10. De igual manera que en el arco circular, así se definen el intradós y extradós del arco apuntado. En este caso se analiza un arco apuntado cuyos semiarcos están determinados por circunferencias únicas.

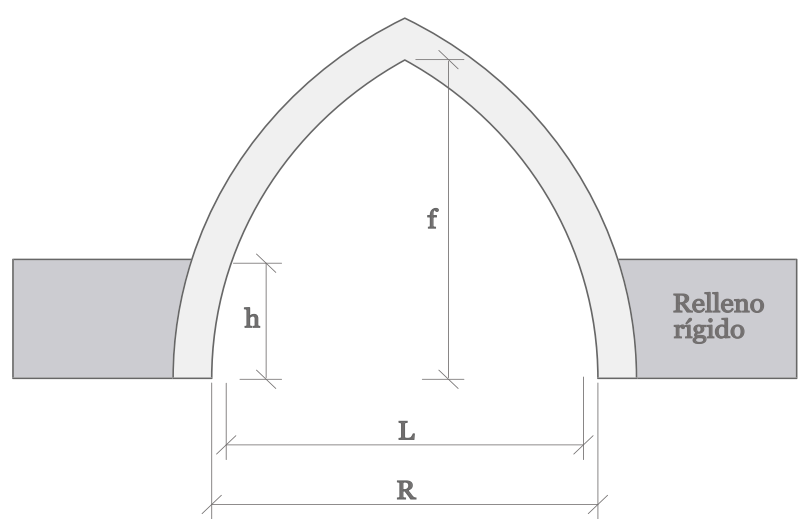

Figura 10. Geometría de arco apuntado.

En la Figura 11, se presenta la evolución de las deformadas para incrementos de desplazamiento impuesto de un estribo. 
En este tipo de geometría, es posible que la línea de empujes sea tangente en dos puntos en el extradós, en la zona de clave, para la hipótesis buscada de empuje mínimo. Si se consideran las dos rótulas, sumando a las dos rótulas del intradós, la estructura estaría ante un mecanismo de colapso al pasar súbitamente de dos a cuatro rótulas, lo que sucedería en la hipótesis más bien poco probable de absoluta simetría de arco, despiece y aparejo de dovelas reales, juntas, ...
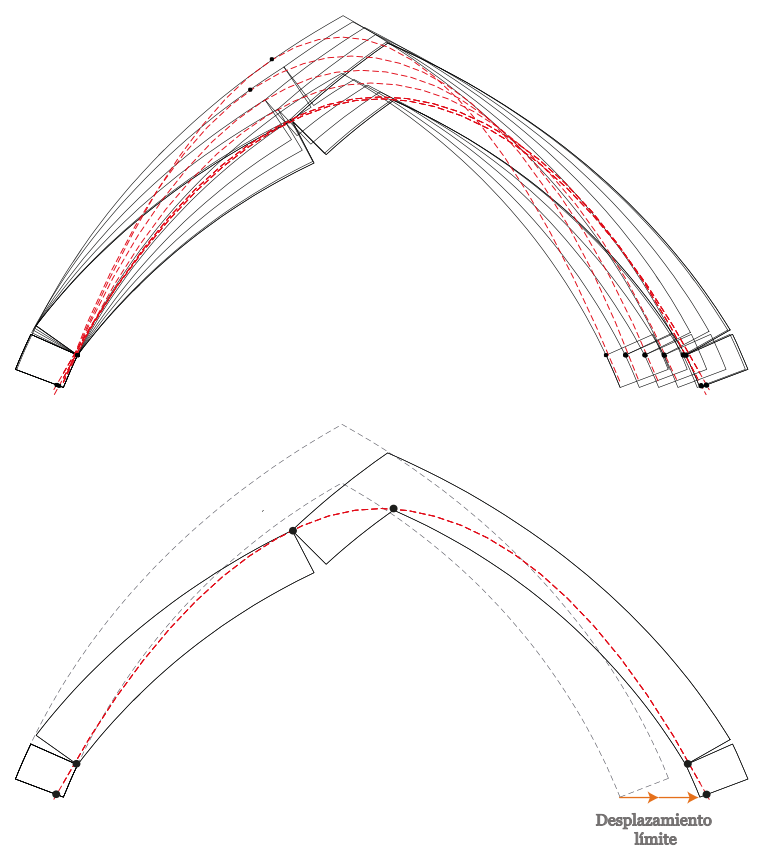

Figura 11. Evolución de la deformada en arcos apuntados.

Por ello, en este análisis ha de considerase una sola rótula, en este caso, por ejemplo (es indiferente) la rótula de la izquierda. Eso es lo que se ha observado en ensayos en laboratorio ( $\mathrm{Fi}$ gura 12), que demuestran por dónde empieza a abrirse el arco cuando existen desplazamientos horizontales. Si el modelo se hubiera construido con un número muy elevado de dovelas, y simetría perfecta (que no existe), deberían apreciarse las dos rótulas teóricas en clave. Cabe recordar que, al tratarse de un problema de equilibrio, un modelo a escala reducida es perfectamente válido como herramienta experimental ya que no existe efecto de escala. Además, el modelo permite observar el cambio de posición de rótulas que experimenta la geometría adaptándose a la nueva condición de contorno conforme aumenta el desplazamiento horizontal. En esta situación, la línea de empujes será tangente en los nuevos puntos.

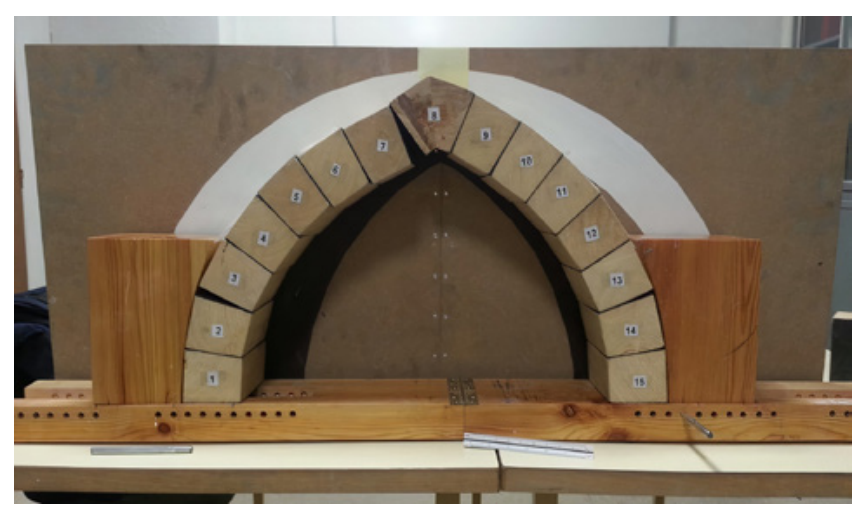

Figura 12. Configuración de rótulas en un arco apuntado al imponer un corrimiento horizontal en el arranque (15).
Aplicando el análisis a varias geometrías de arcos apuntados, definidos por una altura variable de $h$ y con la variación de su canto $e$, se obtienen las curvas de la Figura 13. En ellas se representa el valor de $e / L$ versus el porcentaje de incremento de luz que puede experimentar el arco antes del colapso. En esta parte del análisis se considera únicamente la acción del peso propio.

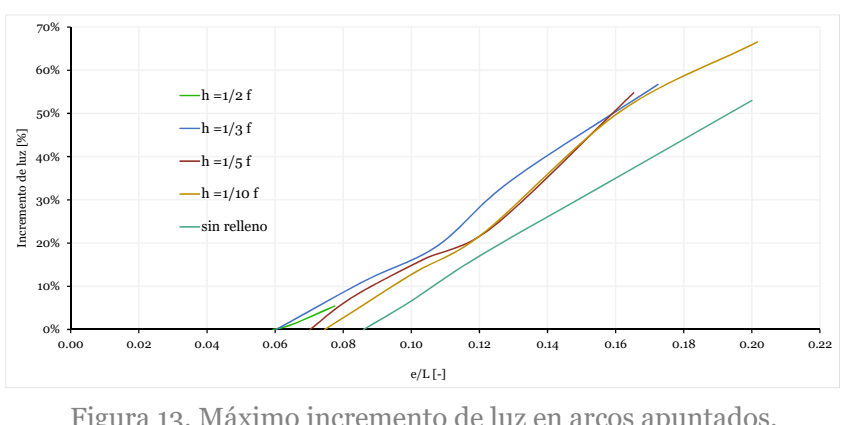

Al comparar los resultados obtenidos para arcos de directriz circular y arcos apuntados, se observa que los arcos apuntados presentan una mayor estabilidad frente a desplazamientos horizontales impuestos, es decir, admiten mayores desplazamientos impuestos a igualdad de luz y de esbeltez. Esto ocurre porque, debido al mecanismo de colapso, el arco siempre termina colapsando hacia el interior y el recorrido cinemático es tanto mayor cuanto mayor sea la flecha. En arcos muy rebajados, donde el espacio a recorrer es menor, el recorrido aceptable es netamente menor. Con los resultados obtenidos a través de la herramienta, y con la gráfica expuesta, es posible aproximar los resultados a ecuaciones con el objetivo de determinar el desplazamiento límite para cualquier relación de $e / L$. Dichas ecuaciones se presentan a continuación para facilitar su digitalización y uso práctico.

Para $h=1 / 2 f$, con valor de $r^{2}=1.00$

$$
\Delta L=77.40(e / L)^{2}+7.60(e / L)-0.1783
$$

Para $h=1 / 3 f$, con valor de $r^{2}=1.00$

$$
\Delta L=8.3904(e / L)^{2}+3.1664(e / L)-0.2246
$$

Para $h=1 / 5 f$, con valor de $r^{2}=0.99$

$$
\Delta L=24.878(e / L)^{2}-0.299(e / L)-0.0873
$$

Para $h=1 / 10 f$, con valor de $r^{2}=0.99$

$$
\Delta L=-2.3422(e / L)^{2}+6.0536(e / L)-0.4474
$$

Sin relleno, con valor de $r^{2}=1.00$

$$
\Delta L=-3.057(e / L)^{2}+5.5213(e / L)-0.4527
$$

\subsection{Arco carpanel}

Como se ha anticipado, la herramienta desarrollada permite deducir el desplazamiento límite de arcos de cualquier geometría. Por ejemplo, se ha analizado un arco carpanel del palacio Carlos $\mathrm{V}$ en Granada. El arco tiene una relación $e / L$ de 0.14 y una relación $f / L$ de 0.29 . La evolución de la deformada hasta encontrar el desplazamiento límite se presenta 
en la Figura 14, dando como resultado un incremento de luz del $11.70 \%$.

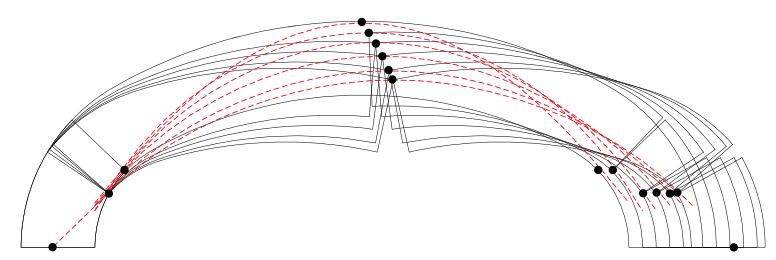

Figura 14. Evolución de la deformada de un arco carpanel.

\section{APLICACIÓN A UN CASO REAL: EL PUENTE MANTIBLE}

\subsection{Descripción}

En este apartado se presenta la aplicación de la metodología a un caso de estudio concreto, el puente Mantible (Logroño), (quizás) romano que, con el paso del tiempo, ha ido perdiendo bóvedas, quedando en pie hoy en día únicamente dos arcos de medio punto (Figura 15). El puente fue declarado Patrimonio Histórico de España el 25 de enero de 1983.

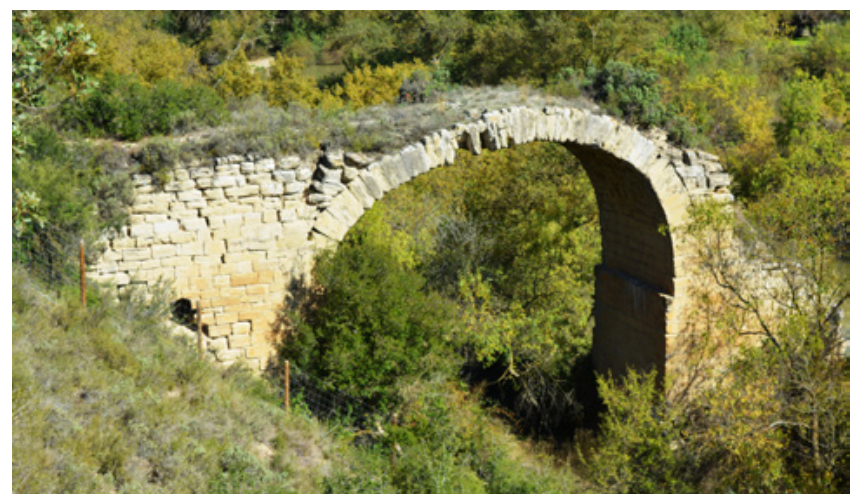

Figura 15. Puente Mantible, Logroño (foto de los autores)

Inspecciones recientes han puesto de manifiesto cómo uno de sus arcos se había desplazado $0.43 \mathrm{~m}$ horizontalmente hacia afuera, debido al giro de la pila situada al borde del cauce, conduciendo a la geometría en el estado actual fuera la que muestra la Figura 16.

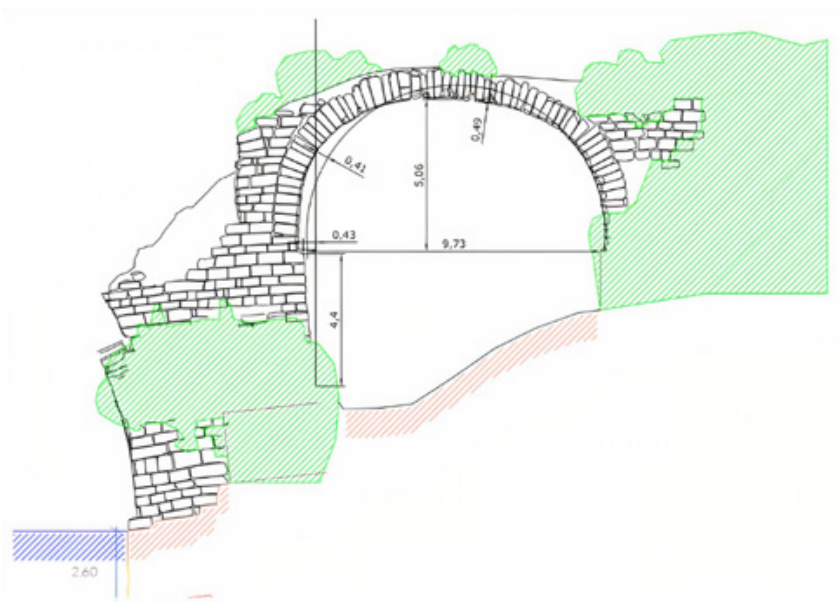

Figura 16. Geometría actual de un arco de puente Mantible, tomada del trabajo de A. Rodríguez y J.M. Valle (Laboratorio de Documentación Geométrica del Patrimonio, Grupo de Investigación en Patrimonio Construido - GPAC (UPV-EHU).
Además del desplazamiento sufrido, debido al paso de los años, la estructura ha perdido material. El origen de estos deterioros, con fisura longitudinal propia de hendimiento por compresión localizada puede asociarse a elevadas tensiones de compresión en ciertas zonas de la bóveda, a la meteorización ambiental o a ambas cosas (Figura 17).

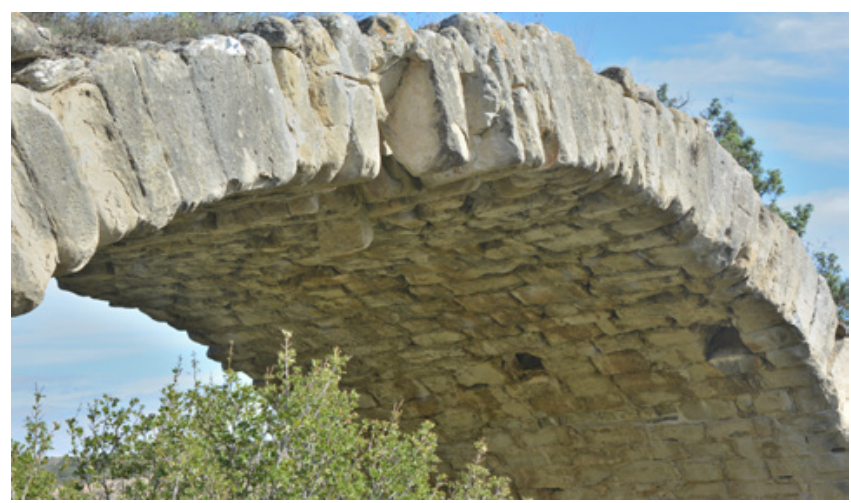

Figura 17. Pérdida y fractura de dovelas (foto de los autores).

Los datos proporcionados para el estudio de este arco de originaria directriz circular son: radio $4.87 \mathrm{~m}$, luz $9.74 \mathrm{~m}$, y espesor de $0.80 \mathrm{~m}$. Por tanto, $e / L=1 / 12$. Estos valores corresponden al estado inicial del arco, es decir, recién construido. Se ha supuesto que el espesor es uniforme en todo el ancho de la bóveda.

\subsection{Análisis paramétrico}

Debido a la evidente pérdida de materiales se ha realizado un análisis paramétrico considerando varios espesores del arco para estimar su influencia en el desplazamiento límite de la pila. Se considera el arco trabajando sólo a peso propio ya que, de acuerdo con los levantamientos previos que se han realizado, existe un desprendimiento del relleno rígido cuyo aporte actual es casi nulo. A ese respecto hay que añadir que, a diferencia del relleno suelto o granular, el rígido se separa del trasdós de la bóveda y, por tanto, no colabora allí donde se despega, como se ha anticipado más arriba, en este mismo artículo.

\subsection{Resultados}

Si no se considerara la pérdida de mortero, es decir, si no se redujera el espesor de $0.75 \mathrm{~m}$, el valor del desplazamiento límite obtenido con la herramienta es de $0.76 \mathrm{~m}$ (7.8\% de la luz primitiva). Considerando que la estructura ya ha sufrido un desplazamiento de $0,43 \mathrm{~m}$, se deduce que quedarían $0.33 \mathrm{~m}$ hasta alcanzar el colapso. En la tabla 1 se muestran los valores máximos obtenidos variando el espesor de la pieza. Una reducción del $7 \%$ del espesor lleva una reducción del desplazamiento límite del 26\%.

Tabla 1. Desplazamiento límite para diferentes espesores

\begin{tabular}{|c|c|}
\hline Espesor (m) & Desplazamiento límite (m) \\
\hline 0.70 & 0.56 \\
\hline 0.75 & 0.76 \\
\hline 0.85 & 0.95 \\
\hline 0.90 & 1.20 \\
\hline
\end{tabular}

En la Figura 18 se presentan las secuencias de deformadas del arco debidas a unos desplazamientos impuestos horizontales hacia el exterior, hasta alcanzar el colapso. 


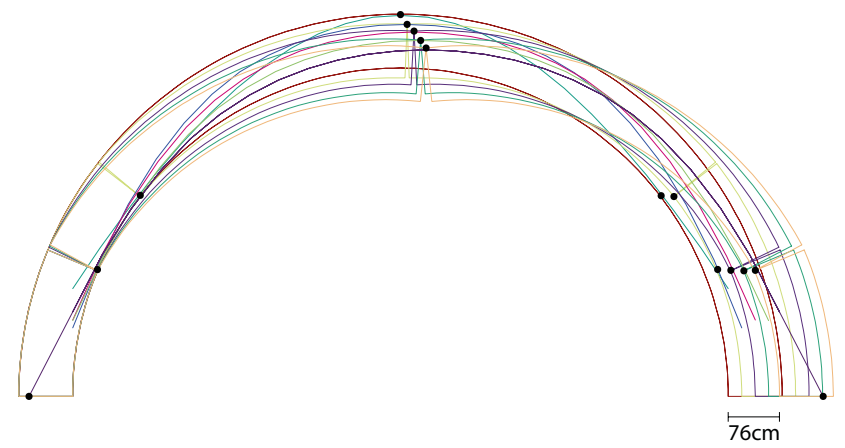

Figura 18. Evolución de deformada del arco de puente de Mantible.

El incremento de desplazamiento impuesto lleva implícito una reducción de la flecha que, a su vez, genera un incremento del empuje horizontal. El arco en su estado inicial, cargado bajo su propio peso, moviliza un empuje horizontal de $47.26 \mathrm{kN}$. La figura 19 presenta la evolución (relativa con respecto al empuje para desplazamiento nulo) de los empujes

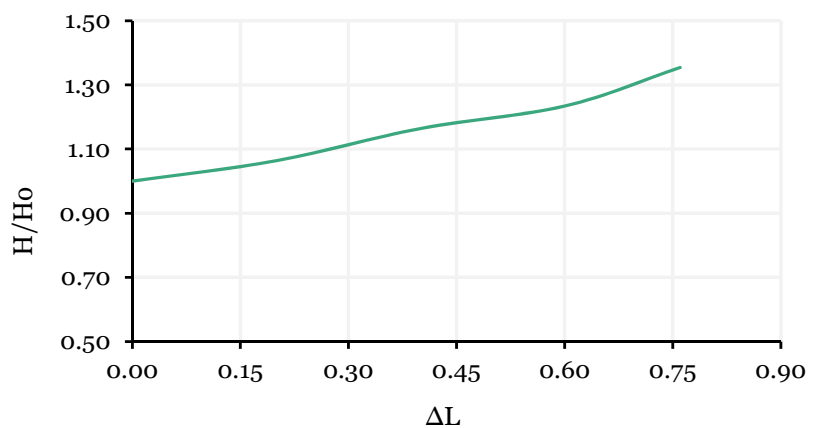

Figura 19. Incremento del empuje horizontal para valores crecientes del desplazamiento impuesto. horizontales para diferentes valores de desplazamiento impuesto, hasta alcanzar al colapso. La velocidad de crecimiento del empuje no es monótona debido a la no linealidad del problema y al cambio de posición de las rótulas (figura 18). $\mathrm{Al}$ producirse el colapso, el arco habrá incrementado la componente horizontal de la reacción en un 35\%, manteniéndose constante, lógicamente, la vertical. Dicho análisis es determinante para estudiar un posible vuelco de la pila sobre la que apoya la bóveda.

\section{CONCLUSIONES}

En este artículo se ha presentado una metodología y su implementación en una herramienta paramétrica para determinar el desplazamiento límite horizontal de arcos de cualquier geometría, cargados bajo su peso propio, con o sin relleno granular. Los arcos de directriz circular han sido estudiados bajo la presencia de un relleno granular, demostrando el gran aporte que significa para la estabilidad y resistencia de los arcos de fábrica.

Finalmente, las gráficas proporcionadas resultan de gran utilidad en la praxis profesional sin la necesidad de acudir a análisis no lineales. En definitiva, se solventa un problema práctico que no estaba resuelto, ayudando a los técnicos encargados de la conservación del patrimonio a tomar las decisiones más oportunas.

Las futuras líneas de investigación irán enfocadas a estudiar no sólo el desplazamiento horizontal límite, sino también desplazamientos verticales y la combinación de ambos, incorporándolos a la herramienta ya creada. Del mismo modo, sería muy interesante estudiar los desplazamientos impuestos hacia el interior (algo quizás menos frecuente y de menores consecuencias), generando un incremento de la flecha, lo cual conlleva una disminución de los empujes horizontales.

\section{REFERENCIAS}

(1) Ochsendorf, J. (2002). Collapse of masonry structures (Tesis Doctoral). University of Cambridge. Recuperado de http://www.repository.cam.ac.uk/handle/1810/244820.

(2) Heyman, J. (1966). The stone skeleton. International Journal of Solid and Structures, 2(2): 249-279. http://dx.doi. org/10.1016/0020-7683(66)90018-7.

(3) Huerta Fernández S. (2004). Arcos, bóvedas y cúpulas: geometría y equilibrio en el cálculo tradicional de estructuras de fábrica. Madrid: Instituto Juan de Herrera.

(4) Todisco, L., León, J. (2017). Criterios de análisis y comprobación de estructuras de fábrica. En: J.M. Goicolea, J. León (Eds.), Los Puentes Piedra (o Ladrillo) Antaño y Hogaño (pp. 158-168). Madrid: Fundación Juanelo Turriano.

(5) Martínez Martínez, J.L. (2003). Determinación teórica y experimental de diagramas de interacción de esfuerzos en estructuras de fábrica y aplicación al análisis de construcciones históricas (Tesis doctoral). Universidad Politécnica de Madrid.

(6) Mann, W., Muller, H. (1982). Failure of shear-stressed masonry - An enlarged theory, tests and application to shearwalls, Proceedings of the British Ceramic Society. 30(1): 233-235.

(7) Coccia, S., Di Carlo, F., Rinaldi Z. (2015). Collapse displacements for a mechanism of spreading-induced supports in a masonry arch. International Journal of Advanced Structural Engineering, 3(7): 307-320. https://doi.org/10.1007/ s40091-015-0101-x.

(8) Di Carlo, F., Coccia, S., Rinaldi, Z. (2018). Collapse load of a masonry arch after actual displacements of the supports. Archive of Applied Mechanics, 88, 1545-1558. https://link.springer.com/10.1007/so0419-018-1386-6.

(9) Di Carlo, F., Coccia, S. (2020). Collapse state of elliptical masonry arches after finite displacements of the supports. Engineering Failure Analysis. 114, 104593. https://doi.org/10.1016/j.engfailanal.2020.104593.

(10) Galassi, S., Misseri, G., Rovero, L., Tempesta, G. (2020). Analysis of masonry pointed arches on moving supports: A numeric predictive model and experimental evaluations. En: Lecture Notes in Mechanical Engineering (pp. 2048-2068). Netherlands: Springer. https://link.springer.com/10.1007/978-3-030-41057-5_163.

(11) Zampieri, P., Amoroso, M., Pellegrino, C. (2019). The masonry buttressed arch on spreading support. Structures, 20, 226-236. https://doi.org/10.1016/j.istruc.2019.03.008.

(12) Intrigila, C., Nodargi, N.A., Bisegna, P. (2019). Square Cross Vaults on Spreading Supports. En: RILEM Bookseries (pp. 1045-1053). Netherlands: Springer. https://link.springer.com/10.1007/978-3-319-99441-3_113. 
(13) Galassi, S., Misseri, G., Rovero, L., Tempesta, G. (2018). Failure modes prediction of masonry voussoir arches on moving supports. Engineering Structures. 173, 706-717. https://doi.org/10.1016/j.engstruct.2018.07.015.

(14) Zampieri, P., Faleschini, F. Zanini, M.A., Simoncello, N. (2018). Collapse mechanisms of masonry arches with settled springing. Engineering Structures, 156, 363-374. https://doi.org/10.1016/j.engstruct.2017.11.048.

(15) Barba, J. (2018). Vertical Limit Displacements of Masonry Structures (TFM). Universidad Politécnica de Madrid.

(16) Masciotta, M.G., Brigante, D., Barontini, A., Pellegrini, D., Lourenço, P.B., Fabbrocino, G., Padovani, C., Girardi, M. (2020) Experimental and Numerical Investigations of a Segmental Masonry Arch Subjected to Horizontal Settlements. En: Proceedings of ARCH 2019 (pp. 413-421). https://doi.org/10.1007/978-3-030-29227-0_43.

(17) Acikgoz, S., Soga, K. , Woodhams, J. (2017). Evaluation of the response of a vaulted masonry structure to differential settlements using point cloud data and limit analyses. Construction and Building Materials, 150, 916-931. https://doi. org/10.1016/j.conbuildmat.2017.05.075.

(18) Mutiloa Albizuri L. (2018). Desplazamientos límite en estructuras de fábrica: aproximación teórica y desarrollo de una herramienta (Trabajo Fin de Máster). Universidad Politécnica de Madrid. Recuperado de http://oa.upm.es/52446/.

(19) Ramos, A. (2015). Caracterización estructural de los rellenos situados en el trasdós de bóvedas de edificios históricos (Tesis Doctoral). Universidad Politécnica de Madrid. Recuperado de http://oa.upm.es/38758/

(20) McNeel, R. (2020). Grasshopper generative modeling for Rhino. Computer software. http://www.grasshopper3d.com/.

(21) McNeel, R. (2020). Rhinoceros NURBS modeling for Windows. Computer software. http://www.rhino3d.com/.

(22) Autodesk (2020). AutoCad. https://www.autodesk.es/products/autocad/.

(23) Ramos, A., León, J. (2013). Clasificación morfológica de los rellenos en el trasdós de bóvedas de fábrica, Informes de la Construcción. 65(532), 471-480. doi: https://doi.org/10.3989/ic.12.062. 